\title{
ПАРАМЕТРИЧЕСКАЯ ИДЕНТИФИКАЦИЯ ТИПОВЫХ СПЕКТРАЛЬНЫХ ПЛОТНОСТЕЙ СЛУЧАЙНЫХ ПРОЦЕССОВ ОЦЕНИВАНИЕМ ИХ СРЕДНЕКВАДРАТИЧЕСКИХ ЧАСТОТ
}

\author{
Хобин В. А. ${ }^{1}$, Лагерная С. И. ${ }^{1}$ \\ ${ }^{1}$ Одесская национальная академия пищевых технологий, Одесса \\ Email: svetlanalagernaya@yandex.ru
}

Copyright (C) 2014 by author and the journal “Automation technological and business - processes”. This work is licensed under the Creative Commons Attribution International License (CC BY). http://creativecommons.org/licenses/by/4.0/

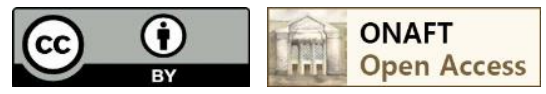

\begin{abstract}
Аннотация
В статье рассматривается метод оценивания параметров случайных процессов, который не требует применения процедуры параметрической оптимизации, что упрощает его использование в реальном времени. Получены аналитические зависимости для оценивания параметров, приведены результаты компьютерного эксперимента, иллюстрирующего эффективность метода.
\end{abstract}

Abstract

In the article the method for estimation of stochastic processes parameters, which does not require the procedure of parametric optimization and can be used in the real time is proposed. The analytical expressions for parameters estimating and results of computer-experiment illustrating the effectiveness of the method are obtained.

\section{Ключевые слова}

Случайный процесс, спектральная плотность, среднеквадратическая частота, оценки, параметрическая идентификация

Технологические процессы в пищевой промышленности как объекты управления характеризуются высокой нестационарностью их свойств, обусловленной большим количеством факторов (характеристики сырьевых и энергетических потоков, состояние рабочих органов и активных зон технологических агрегатов) существенно влияющих на работу технологических агрегатов, но практически недоступных для измерения. Повышение эффективности управления такими объектами может быть достигнуто, например, использованием адаптивных систем. Для создания таких систем автоматического управления требуется получать информацию об изменениях характера функционирования объектов. Практика показывает, что управляемые переменные корректно могут рассматриваться, как случайные процессы, поэтому информация об изменении должна быть связана с оценкой вероятностных характеристик этих процессов, в частности их спектральных плотностей или корреляционных функций.

Анализ случайных процессов является развивающимся направлением в различных науках. Начиная с середины 60-х годов, в связи с появлением и развитием ЭВМ, интерес к подобной проблематике взрастает. Можно выделить два подхода к решению задач идентификации случайных процессов. Первый - использует для 


\section{I ПИТАННЯ ТЕОРІЇ, МЕТОДИ ТА АЛГОРИТМИ ЕФЕКТИВНОГО АВТОМАТИЧНОГО} УПРАВЛІННЯ ОБ'ЄКТАМИ ХІМІКО-ТЕХНОЛОГІЧНОГО ТИПУ

получения моделей зарегистрированную реализацию случайного процесса, второй связан с обработкой случайных процессов в режиме реального времени. Существует множество статистических способов оценки корреляционной функции стационарного случайного процесса. Все они отличаются друг от друга оперативностью, сложностью технической реализации, а также способами представления результатов. Наиболее известным и исторически первым является способ, получаемый непосредственно из определения автокорреляционной функции путем замены оператора математического ожидания на оператор усреднения. Оценка корреляционной функции таким способом требует значительно большей длительности реализации, чем, например, оценка математического ожидания и дисперсии. Способ непосредственной оценки корреляционной функции стационарного случайного процесса целесообразно применять лишь тогда, когда полностью отсутствует априорная информация о корреляционных свойствах анализируемого процесса. В тех же случаях, когда такая информация имеется, больший эффект дает использование аппроксимативных способов оценивания корреляционных функций [1]. Основная идея аппроксимативного способа оценки корреляционных функций заключается в выборе модели нормированной корреляционной функции этого или иного критерия. Наиболее распространенным на практике является способ, основанный на обеспечении минимума величины квадратической погрешности. Этот метод применяется тогда, когда есть, достаточно априорной информации о свойствах исследуемого процесса.[3]

При решении задач самонастройки регуляторов стремятся получить параметры моделей случайных процессов в реальном времени и минимизировать вычислительные ресурсы. Кроме того, отметим, что для подобных задач существует ограниченный набор спектральных плотностей, которые часто называют типовыми.

В статье рассмотрим экономичный вариант оценивания параметров типовых спектральных плотностей (корреляционных функций) случайных процессов, который может быть реализован в реальном времени и, что очень важно, без применения процедуры параметрической оптимизации.

Рассмотрим дифференцируемый случайный процесс $y_{1}(t)$, корреляционная функция $R_{y 1}\left(\tau_{k}\right)$ и спектральная плотность $S_{y 1}(\omega)$ которого могут быть приняты однопараметровыми в виде:

$$
R_{y 1}\left(\tau_{k}\right)=\sigma_{y 1}^{2} e^{-\alpha|\tau k|}\left(1+\alpha\left|\tau_{k}\right|\right) \quad S_{y 1}(\omega)=\sigma_{y 1}^{2} 4 \alpha^{3} /\left(\omega^{2}+\alpha^{2}\right)^{2}
$$

где $\sigma_{y 1}^{2}$ - дисперсия случайного процесса $y_{1}(t)$;

$\alpha$ - коэффициент спада корреляционной функции (параметр, подлежащий идентификации).

Известно, что среднеквадратическая частота $\omega^{\text {скв }}$ дифференцируемого случайного процесса может быть найдена, как отношение среднеквадратических частот дифференцированного процесса $y_{1}^{\prime}(t)$ и процесса $y_{1}(t)$ :

$$
\omega_{y 1}^{c K B}=\sigma_{y 1^{\prime}} / \sigma_{y 1}=\left|\sqrt{\left(\frac{1}{2 \pi} \int_{-\infty}^{\infty} S_{y 1^{\prime}}(\omega) d \omega\right) /\left(\frac{1}{2 \pi} \int_{-\infty}^{\infty} S_{y 1}(\omega) d \omega\right)}\right|
$$

Для интегрирования спектральных плотностей можно использовать табличный интеграл [2].

$$
I_{n}=1 / 2 \pi \int_{-\infty}^{\infty}(G(j \omega) /(A(j \omega) A(-j \omega))) d \omega
$$

где $G(j \omega)=b_{0}(j \omega)^{2 n-2}+b_{1}(j \omega)^{2 n-4}+\ldots+b^{n-1}$;

$A(j \omega)=a_{0}(j \omega)^{n}+a_{1}(j \omega)^{n-1}+\ldots+a_{n}$

Проинтегрируем спектральную плотность (1) процесса $y_{1}(t)$, для чего запишем ее так, чтоб знаменатель был представлен в виде комплексно-сопряженных сомножителей и найдем коэффициенты полиномов $A(j \omega)$ и $G(j \omega)$ :

$$
\begin{gathered}
S_{y 1}(\omega)=\sigma_{y 1}^{2} 4 \alpha^{3} /\left(\left((j \omega)^{2}+2 \alpha(j \omega)+\alpha^{2}\right)\left((j \omega)^{2}-2 \alpha(j \omega)+\alpha^{2}\right)\right), \\
a_{0}=1 ; a_{1}=2 \alpha ; a_{2}=\alpha^{2} ; b_{0}=0 ; b_{1}=\sigma_{y 1}{ }^{2} 4 \alpha^{3} .
\end{gathered}
$$

Запишем табличное выражение интеграла (3) для $n=2$, так как степень полинома $A(j \omega)$ равна двум и, подставив коэффициенты, получим значение интеграла от спектральной плотности процесса $y_{1}(t)$ :

$$
I_{21}=\left(-b_{0}+a_{0} b_{1} / a_{2}\right) / 2 a_{0} a_{1}
$$

$$
I_{21}=\left(\sigma_{y 1}^{2} 4 \alpha^{3} / \alpha^{2}\right) / 4 \alpha=\sigma_{y 1}^{2}
$$




\section{$\underline{1}$ ПИТАННЯ ТЕОРІЇ, МЕТОДИ ТА АЛГОРИТМИ ЕФЕКТИВНОГО АВТОМАТИЧНОГО УПРАВЛІННЯ ОБ'ЄКТАМИ ХІМІКО-ТЕХНОЛОГІЧНОГО ТИПУ}

Спектральная плотность случайного процесса $y_{1}{ }^{\prime}(t)$, полученного дифференцированием исходного процесса $y_{1}(t)$, или, что тоже самое, - прошедшего через дифференцирующее звено с амплитудо-частотной характеристикой $A(\omega)=\omega$, может быть найдена таким образом:

$$
\begin{gathered}
S_{y 1^{\prime}}(\omega)=|A(\omega)|^{2} S_{y 1}(\omega)=\omega^{2} S_{y 1}(\omega) \\
S_{y 1^{\prime}}(\omega)=-\sigma_{y 1}{ }^{2} 4 \alpha^{3}(j \omega)^{2} /\left(\left((j \omega)^{2}+2 \alpha(j \omega)+\alpha^{2}\right)\left((j \omega)^{2}-2 \alpha(j \omega)+\alpha^{2}\right)\right)
\end{gathered}
$$

Интегрирование $S_{y 1}(\omega)$ будем вести аналогично интегрированию $S_{y 1}(\omega)$. Необходимо отметить, что полином знаменателя и значение его коэффициентов при этом останутся прежними (5). Запишем коэффициенты полинома числителя и вычислим значение табличного интеграла (6) для $S_{y 1^{\prime}}(\omega)$ :

$$
\begin{gathered}
b_{0}=-\sigma_{y}^{2} 4 \alpha^{3} ; b_{1}=0 . \\
I_{21}^{\prime}=\sigma_{y}^{2} 4 \alpha^{3} / 4 \alpha=\sigma_{y}^{2} \alpha^{2} .
\end{gathered}
$$

Проинтегрировав спектральные плотности, можно вычислить значение среднеквадратической частоты для случайного процесса с характеристиками (1):

$$
\omega_{y 1}^{c \kappa \beta}=\sigma_{y 1^{\prime}} / \sigma_{y 1}=\left|\sqrt{\sigma_{y 1}^{2} \alpha^{2} / \sigma_{y 1}^{2}}\right|=\alpha .
$$

Учитывая (11), можно записать выражение для оценки параметра $\alpha$ по результатам оценивания среднеквадратической частоты случайного процесса $y_{1}(t)$ :

$$
\hat{\alpha}=\hat{\omega}_{y 1}^{c \kappa B}=\hat{\sigma}_{y 1^{\prime}} / \hat{\sigma}_{y 1} \text {. }
$$

Рассмотрим вариант решение подобной задачи для случайного процесса $y_{2}(t)$ с двухпараметровыми $R_{y 2}\left(\tau_{k}\right)$ и $S_{y 2}(\omega)$ вида:

$$
R_{y 2}\left(\tau_{k}\right)=\sigma_{y 2}^{2} e^{-\alpha|\tau k|}\left(\cos \left(\beta\left|\tau_{k}\right|\right)+(\alpha / \beta) \sin \left(\beta\left|\tau_{k}\right|\right)\right) \quad S_{y 2}(\omega)=\sigma_{y}^{2} 4 \alpha\left(\alpha^{2}+\beta^{2}\right) /\left(\left(\omega^{2}-\beta^{2}-\alpha^{2}\right)^{2}+4 \alpha^{2} \omega^{2}\right),
$$

где $\alpha$ и $\beta$ - параметры характеристик случайного процесса, подлежащие идентификации.

Среднеквадратическая частота случайного процесса с характеристиками (13) может быть найдена по выражению (2). Запишем выражение для спектральных плотностей процессов $y_{2}(t)$ и $y_{2}^{\prime}(t)$ в удобном виде и коэффициенты полиномов числителя и знаменателя:

$$
\begin{gathered}
S_{y 2}(\omega)=\left(\sigma_{y 2}^{2} 4 \alpha\left(\alpha^{2}+\beta^{2}\right)\right) /\left(\left((j \omega)^{2}+2 \alpha(j \omega)+\alpha^{2}+\beta^{2}\right)\left((j \omega)^{2}-2 \alpha(j \omega)+\alpha^{2}+\beta^{2}\right)\right), \\
a_{0}=1 ; a_{1}=2 \alpha ; a_{2}=\alpha^{2}+\beta^{2} ; b_{0}=0 ; b_{1}=\sigma_{y 2}^{2} 4 \alpha\left(\alpha^{2}+\beta^{2}\right), \\
S_{y 2^{\prime}}(\omega)=\left(-\sigma_{y 2}{ }^{2} 4 \alpha\left(\alpha^{2}+\beta^{2}\right)(j \omega)^{2}\right) /\left(\left((j \omega)^{2}+2 \alpha(j \omega)+\alpha^{2}+\beta^{2}\right)\left((j \omega)^{2}-2 \alpha(j \omega)+\alpha^{2}+\beta^{2}\right),\right. \\
a_{0}=1 ; a_{1}=2 \alpha ; a_{2}=\alpha^{2}+\beta^{2} ; b_{0}=-\sigma_{y 2}{ }^{2} 4 \alpha\left(\alpha^{2}+\beta^{2}\right) ; b_{1}=0 .
\end{gathered}
$$

Проведем интегрирование записанных спектральных плотностей, используя выражение табличного интеграла (6), так как степень полинома знаменателя $n=2$.

$$
\begin{gathered}
I_{22}=\left(\sigma_{y 2}{ }^{2} 4 \alpha\left(\alpha^{2}+\beta^{2}\right) / 4 \alpha\right) /\left(\alpha^{2}+\beta^{2}\right) / 4 \alpha=\sigma_{y 2}{ }^{2}, \\
I_{22}{ }^{\prime}=\sigma_{y 2}{ }^{2} 4 \alpha\left(\alpha^{2}+\beta^{2}\right) / 4 \alpha=\sigma_{y 2}{ }^{2}\left(\alpha^{2}+\beta^{2}\right) .
\end{gathered}
$$

Проинтегрировав спектральные плотности случайного процесса с характеристиками (13), получим выражение для среднеквадратической частоты такого процесса.

$$
\omega_{y 2}^{c \kappa \beta}=\sigma_{y 2^{\prime}} / \sigma_{y 2}=\left|\sqrt{\sigma_{y 2}^{2}\left(\alpha^{2}+\beta^{2}\right) / \sigma_{y 2}^{2}}\right|=\left|\sqrt{\left(\alpha^{2}+\beta^{2}\right)}\right|
$$

Выражение (20) можно рассматривать как первое из двух уравнений системы с двумя неизвестными. Для получения второго уравнения рассмотрим прохождение процесса $y_{2}(t)$ с $R_{y 2}\left(\tau_{k}\right)$ и $S_{y 2}(\omega)$ через линейный фильтр с известными параметрами. Переменную на выходе фильтра обозначим $z(t)$. Связь спектральных плотностей на входе $S_{y 2}(\omega)$ и выходе $S_{z}(\omega)$ фильтра определяется известным соотношением:

$$
S_{\mathrm{z}}(\omega)=|W(j \omega)|^{2} S_{y 2}(\omega)=A^{2}(\omega) S_{y 2}(\omega) .
$$

Тогда, если в качестве фильтра выбрать статическое апериодическое инерционное звено 1-го порядка, частотная передаточная функция которого имеет вид $W(j \omega)=1 /(1+j \omega T)$, то $A^{2}(\omega)=1 /\left(1+\omega^{2} T^{2}\right)^{1 / 2}$. Запишем 


\section{1 ПИТАННЯ ТЕОРІї, МЕТОДИ ТА АЛГОРИТМИ ЕФЕКТИВНОГО АВТОМАТИЧНОГО
УПРАВЛІННЯ ОБ'ЄКТАМИ ХІМІКО-ТЕХНОЛОГЧНОГО ТИПУ}

спектральную плотность процесса на выходе фильтра и найдем коэффициенты полиномов числителя и знаменателя для ее интегрирования:

$$
\begin{gathered}
S_{\mathrm{z}}(\omega)=\left((1 /((1+T j \omega)(1-T j \omega))) \cdot\left(\sigma_{y 2}{ }^{2} 4 \alpha\left(\alpha^{2}+\beta^{2}\right)\right)\right) /\left(\left((j \omega)^{2}+2 \alpha(j \omega)+\alpha^{2}+\beta^{2}\right)\left((j \omega)^{2}-2 \alpha(j \omega)+\alpha^{2}+\beta^{2}\right)\right), \\
S_{\mathrm{z}}(\omega)=\left(\sigma_{y 2}{ }^{2} 4 \alpha\left(\alpha^{2}+\beta^{2}\right)\right) /\left(T(j \omega)^{3}+(2 \alpha T+1)(j \omega)^{2}+\left(2 \alpha+T\left(\alpha^{2}+\beta^{2}\right)\right)(j \omega)+\alpha^{2}+\beta^{2}\right), \\
a_{0}=T ; a_{1}=2 \alpha T+1 ; a_{2}=2 \alpha+T\left(\alpha^{2}+\beta^{2}\right) ; a_{3}=\alpha^{2}+\beta^{2} ; b_{0}=0 ; b_{1}=0 ; b_{2}=\sigma_{y 2}{ }^{2} 4 \alpha\left(\alpha^{2}+\beta^{2}\right) .
\end{gathered}
$$

Так как порядок полинома знаменателя $n=3$, то выражение для интеграла от спектральной плотности процесса $z(t)$ будет следующее:

$$
\begin{gathered}
I_{32}=\left(-a_{2} b_{0}+a_{0} b_{1}-a_{0} a_{1} b_{2} / a_{3}\right) / 2 a_{0}\left(a_{0} a_{3}-a_{1} a_{2}\right) \\
I_{32}=\left(\sigma_{y 2}^{2} 2 \alpha(2 \alpha T+1)\right) /\left(2 T^{2} \alpha^{3}+2 T^{2} \alpha \beta^{2}+4 T \alpha^{2}+2 \alpha\right)
\end{gathered}
$$

Запишем выражение для спектральной плотности $S_{z^{\prime}}(\omega)$ процесса $z^{\prime}(t)$, найдем коэффициенты полиномов ее числителя и знаменателя и проинтегрируем ее.

$$
\begin{gathered}
S_{\mathrm{z}^{\prime}}(\omega)=\left(-\sigma_{y 2}{ }^{2} 4 \alpha\left(\alpha^{2}+\beta^{2}\right)(j \omega)^{2}\right) /\left(T(j \omega)^{3}+(2 \alpha T+1)(j \omega)^{2}+\left(2 \alpha+T\left(\alpha^{2}+\beta^{2}\right)\right)(j \omega)+\alpha^{2}+\beta^{2}\right), \\
a_{0}=T ; a_{1}=2 \alpha T+1 ; a_{2}=2 \alpha+T\left(\alpha^{2}+\beta^{2}\right) ; a_{3}=\alpha^{2}+\beta^{2} ; b_{0}=0 ; b_{1}=-\sigma_{y 2}{ }^{2} 4 \alpha\left(\alpha^{2}+\beta^{2}\right) ; b_{2}=0 . \\
I_{32}^{\prime}=\left(\sigma_{y 2}{ }^{2} 2 \alpha\left(\alpha^{2}+\beta^{2}\right)\right) /\left(2 T^{2} \alpha^{3}+2 T^{2} \alpha \beta^{2}+4 T \alpha^{2}+2 \alpha\right)
\end{gathered}
$$

Получим выражение для расчета среднеквадратической частоты процесса $z(t)$ после линейного фильтра:

$$
\omega_{z}^{c \kappa \beta}=\sigma_{z^{\prime}} / \sigma_{z}=\left|\sqrt{\left(\alpha^{2}+\beta^{2}\right) /(2 \alpha T+1)}\right|
$$

Решив систему уравнений (20) и (26), получим выражения для независимого оценивания параметров характеристик случайного процесса с характеристиками (13):

$$
\begin{aligned}
& \left\{\begin{array} { l } 
{ \omega _ { y 2 } ^ { c \kappa в } = \sqrt { ( \alpha ^ { 2 } + \beta ^ { 2 } ) } } \\
{ \omega _ { z } ^ { c \kappa \beta } = \sqrt { ( \alpha ^ { 2 } + \beta ^ { 2 } ) / ( 2 \alpha T + 1 ) } }
\end{array} \Rightarrow \left\{\begin{array}{l}
\left(\omega_{y 2}^{c \kappa в} / \omega_{z}^{c \kappa \beta}\right)^{2}=\left(\alpha^{2}+\beta^{2}\right) /\left(\left(\alpha^{2}+\beta^{2}\right) /(2 \alpha T+1)\right) \\
\beta^{2}=\left(\omega_{y 2}^{c k \beta}\right)^{2}-\alpha^{2}
\end{array} \Rightarrow\right.\right. \\
& \Rightarrow\left\{\begin{array}{l}
\hat{\alpha}=\left(\left(\hat{\omega}_{y 2}^{c \kappa в} / \hat{\omega}_{z}^{c \kappa \beta}\right)^{2}-1\right) / 2 T \\
\hat{\beta}=\sqrt{\left(\hat{\omega}_{y 2}^{c k \beta}\right)^{2}-\left(\left(\left(\hat{\omega}_{y 2}^{c k в} / \hat{\omega}_{z}^{c k \beta}\right)^{2}-1\right) / 2 T\right)^{2}}
\end{array}\right.
\end{aligned}
$$

Необходимо отметить, что оценивание среднеквадратических частот может вестись как по отношению среднеквадратических отклонений (2), так и по формуле:

$$
\hat{\omega}^{c \kappa B}=\pi \hat{s}_{0} / \hat{T}_{p}
$$

где $s_{0}$ - количество пересечений случайным процессом его средней линии на времени $T_{p}$.

Применимость полученных выражений (12) и (28) для оценивания параметров характеристик случайных процессов в реальном времени проверялась в ходе компьютерного эксперимента. При этом тестовые случайные процессы $y_{1}(t)$ и $y_{2}(t)$ генерировались пропусканием белого шума через линейное звено (формирующий фильтр). Для получения процесса с характеристиками (1) использовался формирующий фильтр вида:

$$
W_{\phi \phi 1}(p)=\frac{\left(2 \sigma_{y} \sqrt{\alpha}\right) / \alpha^{2}}{((1 / \alpha) p+1)^{2}},
$$

где $\alpha=1$.

Процесс с характеристиками (13) генерировался с использованием формирующего фильтра

$$
W_{\phi \phi 2}(\mathrm{p})=\frac{2 \sqrt{\sigma_{y}^{2} \alpha\left(\alpha^{2}+\beta^{2}\right)}}{p^{2}+2 \alpha p+\alpha^{2}+\beta^{2}},
$$

где $\alpha=1, \beta=3$.

Оценка среднеквадратических частот случайных процессов велась на скользящих интервалах времени. 


\section{$\underline{1}$ ПИТАННЯ ТЕОРІЇ, МЕТОДИ ТА АЛГОРИТМИ ЕФЕКТИВНОГО АВТОМАТИЧНОГО УПРАВЛІННЯ ОБ'ЄКТАМИ ХІМІКО-ТЕХНОЛОГІЧНОГО ТИПУ}

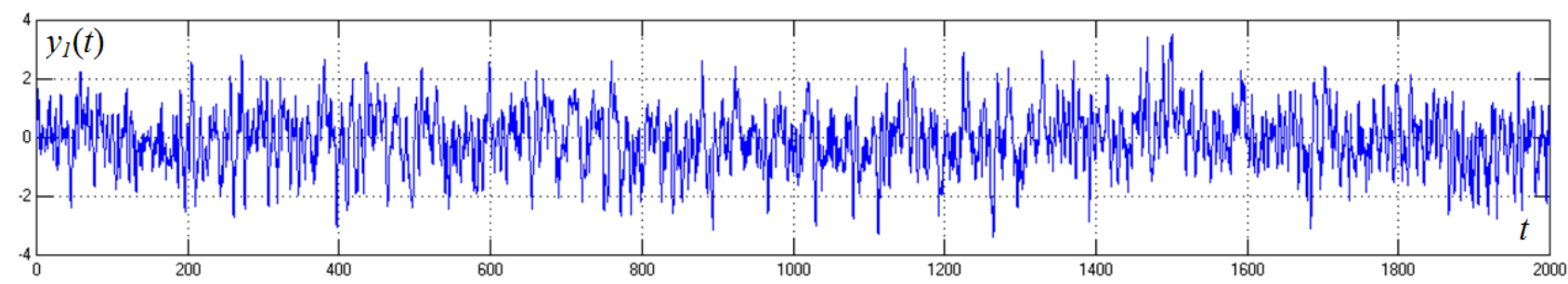

Рис. 1 - Реализация случайного процесса с характеристиками (1)

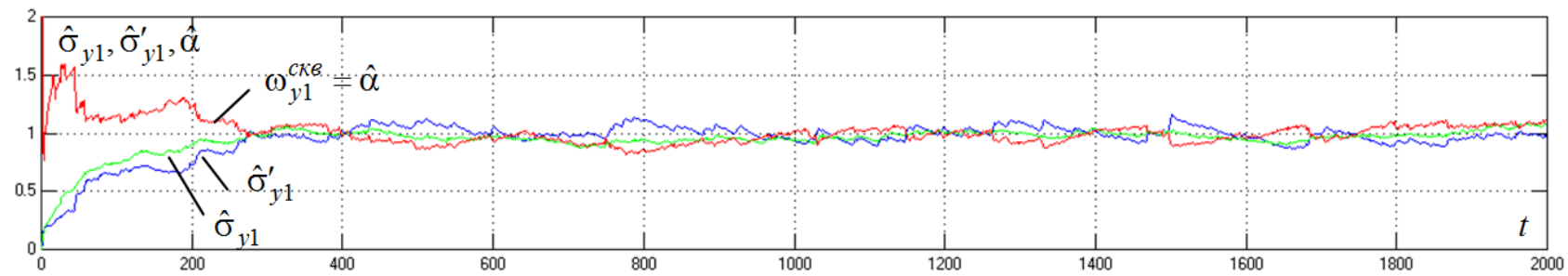

Рис. 2 - Оценки случайного процесса с однопараметровыми характеристиками (1), полученные на скользящих интервалах времени (Тоср=200)

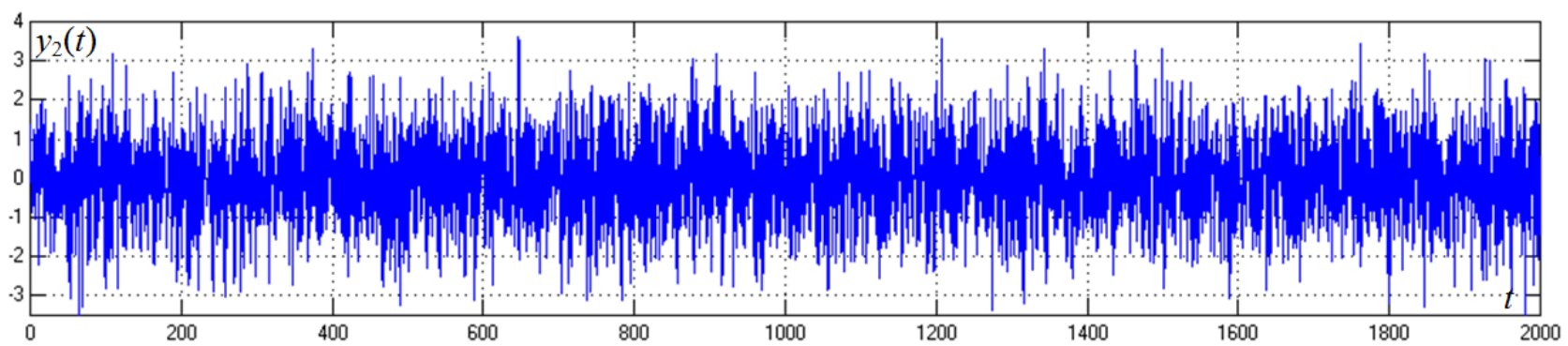

Рис.3 - Реализация случайного процесса с двухпараметровыми характеристиками (13)

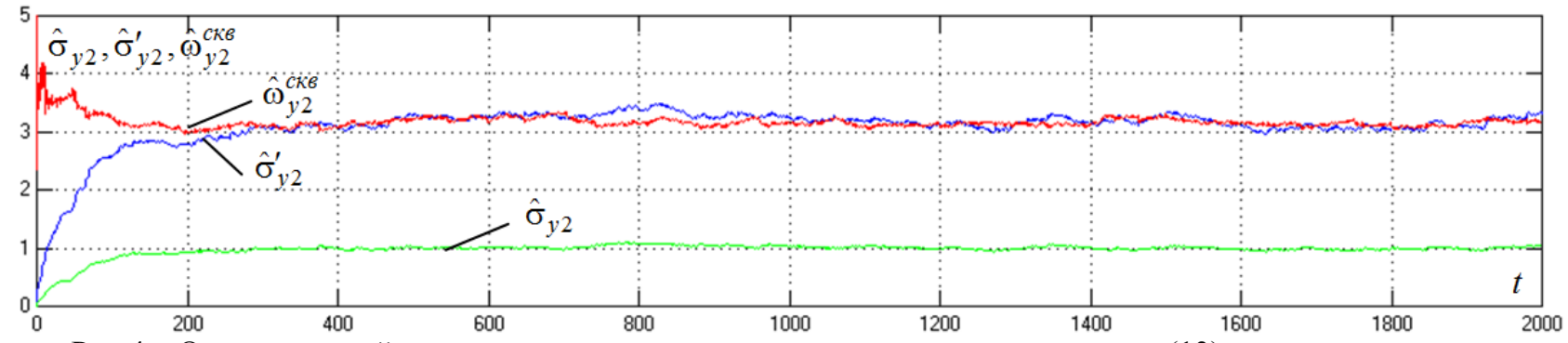

Рис.4 - Оценки случайного процесса с двухпараметровыми характеристиками (13), полученными на скользящих интервалах времени (Тоср=200)

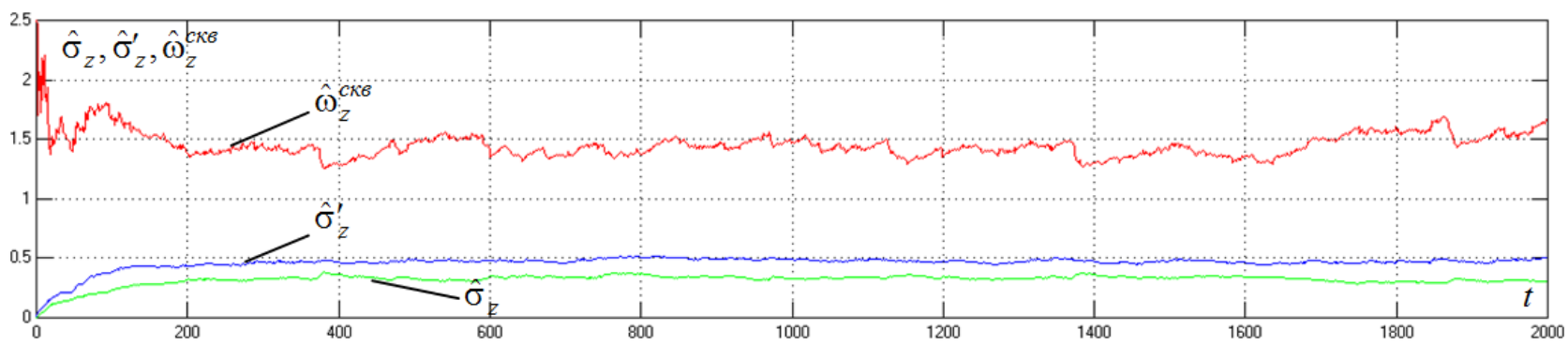

Рис.5 - Оценки случайного процесса $z(t)$ после линейного фильтра, полученные на скользящих интервалах времени $($ Тоср$=200)$ 


\section{1 ПИТАННЯ ТЕОРІЇ, МЕТОДИ ТА АЛГОРИТМИ ЕФЕКТИВНОГО АВТОМАТИЧНОГО} УПРАВЛІННЯ ОБ'ЄКТАМИ ХІМІКО-ТЕХНОЛОГІЧНОГО ТИПУ

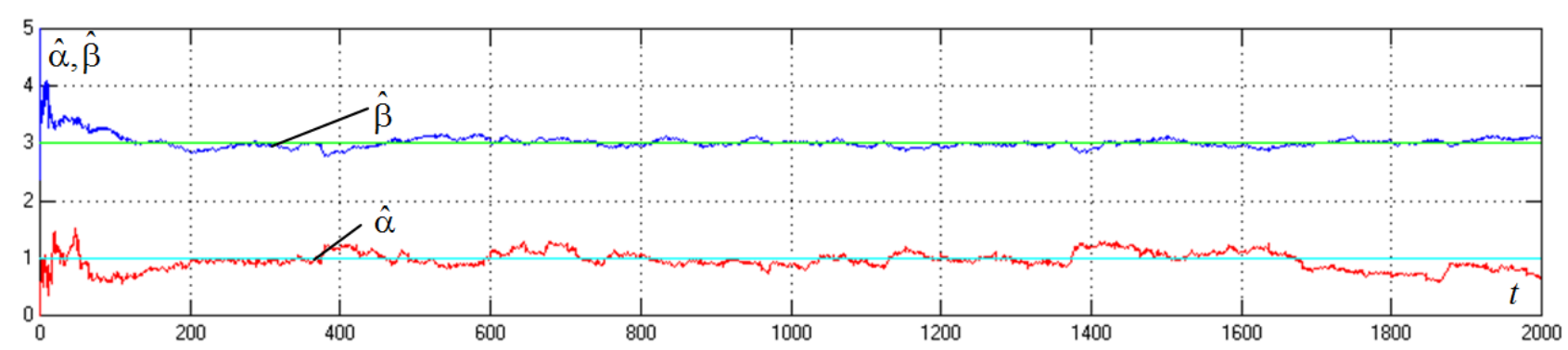

Рис.6 - Оценки параметров спектральной плотности случайного процесса с характеристиками (13), полученные на скользящих интервалах времени (Тоср=200)

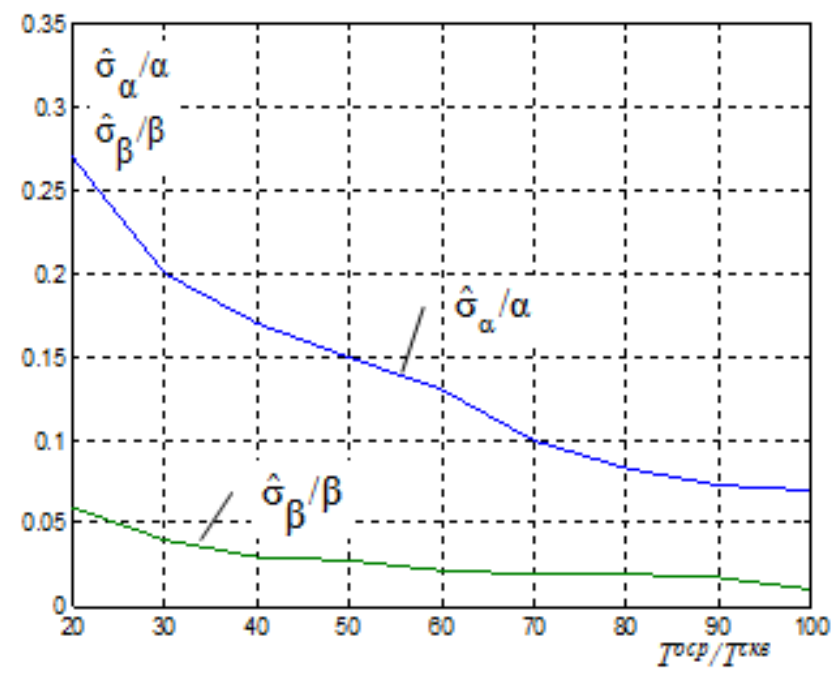

Рис.7 - Влияние относительного интервала осреднения при оценке процессов на долю случайных составляющих в оценках $\hat{\alpha}, \hat{\beta}$

\section{Выводы}

1. Результаты исследований показали, что полученные зависимости (11) и (28) можно использовать для параметрической идентификации на скользящих интервалах времени корреляционных функций и спектральных плотностей случайных процессов с соответствующими характеристиками. Причем, с увеличением интервала осреднения при оценивании случайных процессов, доля случайных составляющих в оценках параметров корреляционных функций ниже.

2.Увеличивая размерность или количество линейных фильтров можно увеличивать количество идентифицируемых параметров, тем самым расширять набор корреляционных функций и спектральных плотностей, для которых можно реализовать аналогичную процедуру идентификации.

3. Перспективами развития данных исследований является нахождение и проверка аналитических выражений для оценивания параметров моделей спектральных плотностей, которыми могут характеризоваться случайные процессы изменения регулируемых переменных в САР. При реализации подобного варианта идентификации параметров случайных процессов в реальном времени, будет получена возможность усовершенствования систем автоматической адаптации.

\section{Литература}

1. Пугачев, В.С. Основы статистической теории автоматических систем [Текст] / В.С. Пугачев, И.Е. Казаков, Л.Г. Евланов. - М., «Машиностроение», 1974. - 400 с.

2. Бесекерский, В.А. Теория систем автоматического управления [Текст] / В.А. Бесекерский, Е.П. Попов // Изд. 4-е, переработанное и доп.- СПб., Профессия, 2007. - 725 с.

3. Левин, Б.Р.Вероятностные модели и методы в системах связи и управления [Текст] / Б.Р. Левин, В. Шварц. - М.: Радио и связь, 1985. - 312 с. 


\section{I ПИТАННЯ ТЕОРІЇ, МЕТОДИ ТА АЛГОРИТМИ ЕФЕКТИВНОГО АВТОМАТИЧНОГО УПРАВЛІННЯ ОБ'ЄКТАМИ ХІМІКО-ТЕХНОЛОГІЧНОГО ТИПУ}

4. Бессонов, А.А. Методы и средства идентификации динамических объектов [Текст] / А.А. Бессонов, Ю.В. Загашвили, А.С. Маркелов. - Л.: Энергоатомиздат. Ленингр. отд-ние, 1989.- 280 с.

5. Четвериков, В.Н. Стохастические вычислительные устройства систем моделирования [Текст] / В.Н. Четвериков, Э.А. Баканович. - М.: Машиностроение, 1989. - 272 с.

6. Шалыгин А.С. Прикладные методы статистического моделирования [Текст] / А.С. Шалыгин, Ю.И. Палагин. - Л.: Машиностроение. Ленингр. отд-ние, 1986. - 320 с.

7. Марпл С. Цифровой спектральный анализ и его приложения [Текст] / С. Марпл. - М. Мир, 1990 - 577 с.

8. Андерсон Т. Статистический анализ временных рядов [Текст] / Т. Андерсон. - М.: Мир, 1976.

9. Бендат Дж. Прикладной анализ случайных процессов [Текст] / Дж. Бендат, А. Пирсол. - М.: Мир, 1989.

10. Активная параметрическая идентификация стохастических линейных систем : [монография] / [В. И. Денисов и др.] ; Новосиб. гос. техн. ун-т. - Новосибирск : Изд-во НГТУ, 2009. - 190 с.

11. Огарков М.А. Методы статистического оценивания параметров случайных процессов [Текст] / М.А. Огарков. - М. Энергоатомиздат, 1980. - 208 с.

\section{Е НОВОСТИ АВТОМАТИЗАЦИИ}

В столице Южно африканской республики - в Кейптауне в период с 24 по 29 августа 2014 пройдет очередной (19-ый) конгресс Международной федерации автоматического управления (IFAC - International Federation of Automatic Control)

С тех пор как конгресс был проведен впервые в 1956 в немецком городке Гейдельберге конгрессы проводились регулярно. На той первой конференции 30 участников подписали декларацию, в которой была ясно определена потребность создать Международную организацию автоматического управления. Подписавшиеся обязались уже способствовать формированию национальных организаций, если не существующий в то время.

В конце Гейдельбергской Конференции Временный Комитет был основан под председательством Виктора Бройды (Франция), чтобы спроектировать конституцию для запланированной Международной федерации Автоматического управления.

12 сентября 1957 Первая Генеральная Ассамблея собралась на учредительной встрече в Париже. Делегаты из 18 стран, представляющих их национальные организации, собрались в Conservatoire National des Arts et Métiers под председательством Виктора Бройды. Они голосовали по конституции и Постановлениям органов местной власти; они выбрали первого президента, Гарольда Честната, а также членов Исполнительного совета, и они назначили председателей комитетов. В свое время президентами Международной федерации по автоматическому управлению были представители СССР. Так, в период с 1958 по 1960 г.г. президентом был Александр М. Летовых, а в период с 1987 по 1990 г.г. президентом был Борис Тамма

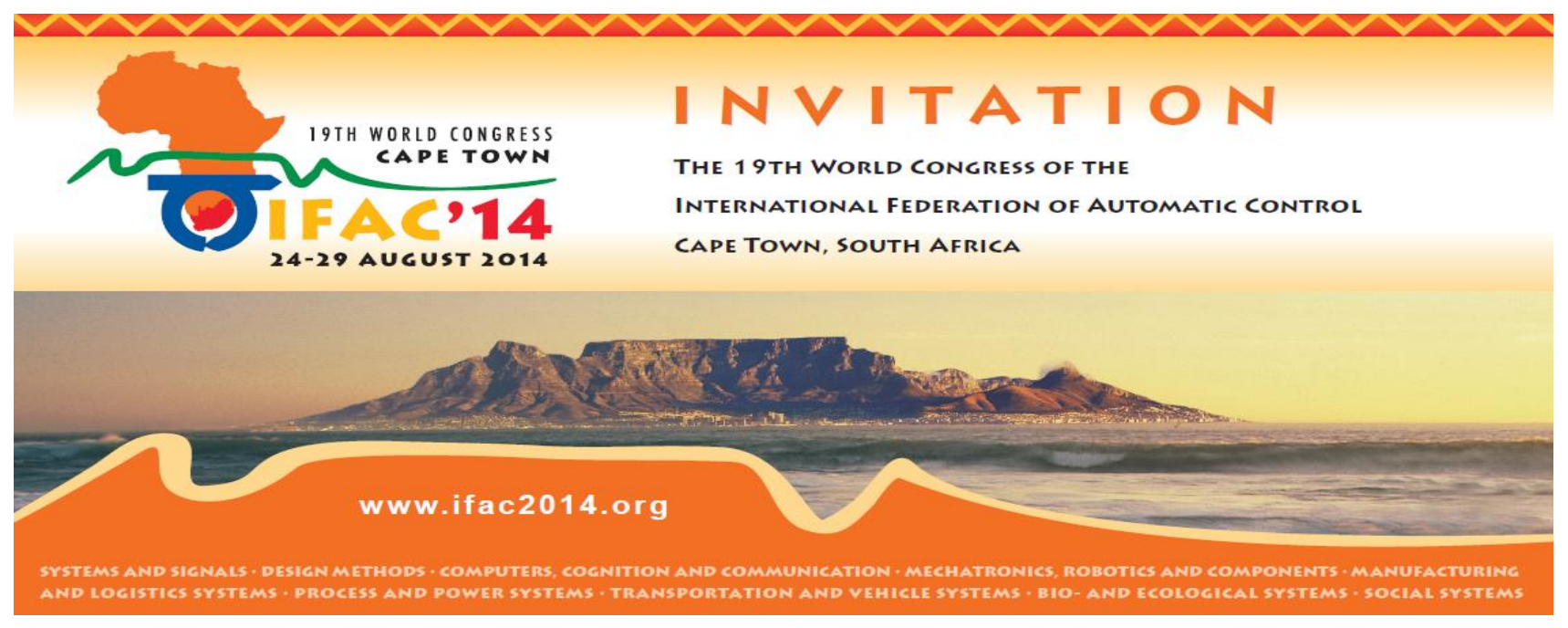

